\title{
Blow-Up and Attractor of Solution for Problems of Nonlinear Schrodinger Equations
}

\author{
Ning Chen*, Jiqian Chen \\ School of Science, Southwest University of Science and Technology, Mianyang, China \\ Email: "chenning783@163.com
}

Received September 18, 2012; revised October 18, 2012; accepted October 26, 2012

\begin{abstract}
In this paper, the authors study the blow-up of solution for a class of nonlinear Schrodinger equation for some initial boundary problem. On the other hand, the authors give out some analyses and that new conclusion by Eigen-function method. In last section, the authors check the nonlinear parameter for light rule power by using of parameter method to get ground state and excite state correspond case, and discuss the global attractor of some fraction order case, and combine numerical test. To illustrate this physics meaning in dimension $d=1,2$ case. So, by numerable solution to give out these wave expression.
\end{abstract}

Keywords: Nonlinear Schrodinger Equation; Eigen-Function Method; Fractional Order; Blow-Up; Glabal Attractor

\section{Introduction}

The quantum mechanics theory and application in more field in nature science. The non-linear Schrodinger equation is the basic equation in nonlinear science and widely applied in natural science such as the physics, chemistry, biology, communication and nonlinear optics etc. (See [1-9]) We study this equation to extend them are with important meaning (See[10-12]).

As we all know, the nonlinear Schrodinger equation be description quantum state of microcosmic grain by wave, it is variable for dependent time, and that is most essential equation, which position and action similarly Newton equation in position and action classics mechanics, it is apply to field as optics, plasma physics, laser gather, cohesions etc, particular on that action of power and trap, search analytical solution for Schrodinger equation is also difficult, and more so difficult for complicated power.

Now, we may extend some results in [4] by using Eigen-function method in through paper.

As we all know the solution of initial problem for Schrodinger equation bellow

$$
\left\{\begin{array}{l}
u_{t}-\mathrm{i} a^{2} \Delta u=f(x, t), x \in R^{n}, t>0, \\
u(x, 0)=\phi(x), x \in R^{n}
\end{array}\right.
$$

Assume that real part and imaginary part of

$$
\phi(x), f(x, t),
$$

are real analytical function for $x \in R^{n}$, then this solution of the problem may expresses in form:

*Corresponding author.

$$
\begin{aligned}
u(x, t) & =\sum_{k=0}^{\infty} \frac{\left(\mathrm{i} a^{2}\right)^{k}}{k !}\left(t^{k} \Delta^{k} \phi(x)\right) \\
& +\int_{0}^{t}(t-\tau)^{k} \Delta_{x}^{k} f(x, \tau) \mathrm{d} \tau .
\end{aligned}
$$

\section{Several Theorems}

In this section, we consider the blow-up of solutions to the mixed problems for higher-order nonlinear Schrodinger equation with as bellow.

It is well known the higher order equation:

$$
u_{t}+\mathrm{i} \Delta^{k} u=f(u), t>0, x \in \Omega,
$$

where

$$
\Delta=\sum_{i=1}^{n} \frac{\partial^{2}}{\partial x_{i}^{2}}, \Delta^{2}=\Delta(\Delta), \cdots, \text { etc. }
$$

that with new results for higher-order case. Now, we consider the blow-up of solutions to the mixed problems for six-order general Schrodinger equation to extend some results [4] that as bellow form:

$$
\left\{\begin{array}{l}
u_{t}+\mathrm{i} \Delta^{3} u=f(u), t>0, x \in \Omega, \\
u(0, x)=\varphi(x), x \in \Omega \\
{\left.\left[\alpha(t, x)\left(\partial \Delta^{2} u / \partial \gamma\right)+\beta(t, x) u\right]\right|_{\partial \Omega}=0, t \geq 0 .}
\end{array}\right.
$$

Assume that

$$
\bar{\lambda} f(\lambda) \geq 0,|f(\lambda)| \geq c|\lambda|^{4 k-1}, \varphi(x) \in H^{6}(\Omega), \varphi(x)
$$


not identical zero.

Where $f$ holds complex value function with selfvariable for complex. $\phi(x)$ is also complex value

$$
\left(\alpha^{2}+\beta^{2} \neq 0,(t, x) \in R^{1} \times R^{n}\right)
$$

Theorem 2.1. Suppose that nonlinear term $f$ of problem (2.1) satisfy $C>0$,

$$
\bar{\lambda} f(\lambda) \geq 0,|f(\lambda)| \geq c|\lambda|^{4 k-1}, \phi(x) \in H^{6}(\Omega),
$$

and $\varphi(x)$ not identical zero then the classical solution of (2.1) must be for blow-up in finite time in

$$
C^{1}\left(0, T ; H^{6}(\Omega)\right) \text {. }
$$

\section{Proof. Let}

$$
J(t)=\int_{\Omega}|u|^{2} \mathrm{~d} x=\int_{\Omega} u \bar{u} \mathrm{~d} x,
$$

Then

$$
\begin{aligned}
& J^{\prime}(t)=\int_{\Omega}\left(u_{t} \bar{u}+u \bar{u}_{i}\right) \mathrm{d} x \\
& =\mathrm{i} \int_{\Omega}\left(u \Delta^{3} \bar{u}-\bar{u} \Delta^{3} u\right) \mathrm{d} x+\int_{\Omega}(u \overline{f(u)}+\bar{u} f(u)) \mathrm{d} x,
\end{aligned}
$$

By the first Green's formula, we have

$\int_{\Omega} u \Delta^{3} \bar{u} \mathrm{~d} x=\int_{\Omega} u\left(\partial \Delta^{2} \bar{u} / \partial \gamma\right) \mathrm{d} s-\int_{\Omega}\left(\sum_{i=1}^{n}\left(u_{x_{i}}\right)\left(\Delta^{2} \bar{u}\right)_{x_{i}}\right) \mathrm{d} x$

$\int_{\Omega} \bar{u} \Delta^{3} u \mathrm{~d} x=\int_{\Omega} \bar{u}\left(\partial \Delta^{2} u / \partial \gamma\right) \mathrm{d} s-\int_{\Omega}\left(\sum_{i=1}^{n}(\bar{u})_{x_{i}}\left(\Delta^{2} u\right)_{x_{i}}\right) \mathrm{d} x$

Substituting it into (2.3), then

$$
\begin{aligned}
J^{\prime}(t) & =i \int_{\Omega}\left(u \frac{\partial \Delta^{3} \bar{u}}{\partial \gamma}-\bar{u} \frac{\partial \Delta^{3} u}{\partial \gamma}\right) \mathrm{d} s \\
& +\int_{\Omega}(u \overline{f(u)}+\bar{u} f(u)) \mathrm{d} x,
\end{aligned}
$$

We may assume $\alpha(t, x) \neq 0$, then we have

$$
\left.\left(u \frac{\partial \Delta^{2} \bar{u}}{\partial \gamma}-\bar{u} \frac{\partial \Delta^{2} u}{\partial \gamma}\right)\right|_{\partial \Omega}=\left.\left(\frac{\beta}{\alpha} u \cdot \bar{u}-\frac{\beta}{\alpha} \bar{u} \cdot u\right)\right|_{\partial \Omega}=0 .
$$

Obviously, from $u \overline{f(u)}+\bar{u} f(u) \geq 2|u| \cdot|f(u)|$, and $|f(u)| \geq c|u|^{4 k-1}$. Therefore, we have

$$
J^{\prime}(t)=\int_{\Omega}(u \overline{f(u)}+\bar{u} f(u)) \mathrm{d} x \geq 2 c \int_{\Omega}|u|^{4 k} \mathrm{~d} x .
$$

By Schwartz inequality:

$$
\left(\int_{\Omega}|u|^{2} \mathrm{~d} x\right)^{2} \leq \int_{\Omega} 1 \mathrm{~d} x \cdot \int_{\Omega}|u|^{4} \mathrm{~d} x \text {, here } c_{1}=\int_{\Omega} \mathrm{d} x>0 .
$$

So,

$$
\int_{\Omega}|u|^{4} \mathrm{~d} x \geq\left(1 / c_{1}\right)\left(\int_{\Omega}|u|^{2} \mathrm{~d} x\right)^{2} .
$$

Inductively, we have

$$
\int_{\Omega}|u|^{12} \mathrm{~d} x \geq\left(1 / c_{1}^{3}\right)\left(\int_{\Omega}|u|^{2} \mathrm{~d} x\right)^{6} \text { etc. }
$$

$$
\int_{\Omega}\left(|u|^{4}\right)^{k} \mathrm{~d} x \geq\left(1 / c_{1}^{k}\right)\left(\int_{\Omega}|u|^{2} \mathrm{~d} x\right)^{2 k}>0 .
$$

Then $J(t)$ increasing function similar in [4] from

$$
\begin{aligned}
J^{\prime}(t) & \geq\left(2 c / c_{1}\right) \int_{\Omega}|u|^{4 k} \mathrm{~d} x \geq\left(2 c / c_{1}^{k}\right)\left(\int_{\Omega}|u|^{2} \mathrm{~d} x\right)^{2 k} \\
& =\left(2 c / c_{1}^{k}\right) J(t)^{2 k}>0,
\end{aligned}
$$

and then there exists $T_{0}<\infty$, such that $\lim _{t \rightarrow T_{0}-} J(t)=\infty$, that is

$$
\lim _{t \rightarrow T_{0}-} \int_{\Omega}|u|^{2} \mathrm{~d} x=\infty .
$$

So, we complete the proof of this Theorem 2.1.

(As positive integer $k=1$, we get it is theorem 3.1 in [4])

Remark. Then we consider that important case is always for the Schrodinger equation may as bellow form

$$
(c>0): u_{t}+\mathrm{i} \Delta^{2} u=c|u|^{2} \cdot u \text {. }
$$

Now, we shall consider also in this similar case:

$$
\left\{\begin{array}{l}
u_{t}+\mathrm{i} \Delta^{3} u=c|u|^{2} \cdot u \\
u(0, x)=\phi(x), x \in \Omega \\
{\left[\alpha(t, x)\left(\partial \Delta^{2} u / \partial \gamma\right)+\beta(t, x) u\right]_{\partial \Omega}=0, t \geq 0 .}
\end{array}\right.
$$

Therefore, we shall obtain the following theorem.

Theorem 2.2. Suppose that non-linear term $f$ of problem (2.1) satisfy $(c>0)$,

$$
\bar{\lambda} f(\lambda) \geq 0,|f(\lambda)| \geq c|\lambda|^{4 k-1},
$$

and

$$
\phi(x) \in H^{6}(\Omega), \varphi(x) \equiv / \equiv 0, \alpha^{2}+\beta^{2} \neq 0,(t, x) \in R^{1} \times R^{n},
$$

then the classical solution of (2.4) must be for blow-up in finite time in $C^{1}\left(0, T ; H^{6}(\Omega)\right)$ (as positive $k=1$, then it is theorem 3.2 in [4]).

Proof. Since $f(u)=c|u|^{2} \cdot u,(c>0)$ satisfies

$$
\bar{\lambda} f(\lambda)=\bar{\lambda} c \lambda|\lambda|^{2}=c|\lambda|^{4 k} \geq 0,
$$

then $|f(\lambda)|=c|\lambda|^{4 k-1}$.

Thus, from theorem 2.1, we complete the proof of theorem 2.2.

Now, we shall give out the following theorem form. Here, we shall consider the problem:

$$
\left\{\begin{array}{l}
u_{t}+\mathrm{i} \Delta^{3} u=F\left(|u|^{2}\right) u, t>0, x \in \Omega, \\
u(0, x)=\phi(x), x \in \Omega, \\
{\left.\left[\alpha(t, x)\left(\partial \Delta^{2} u / \partial \gamma\right)+\beta(t, x) u\right]\right|_{\partial \Omega}=0, t \geq 0 .}
\end{array}\right.
$$

Theorem 2.3 Suppose that non-linear term $f$ of pro- 
blem (2.5) satisfy

$$
(c>0),|F(\lambda)| \geq c|\lambda|^{4 k-3}, \phi(x) \equiv / \equiv 0,
$$

and

$$
\phi(x) \in H^{6}(\Omega), \alpha^{2}+\beta^{2} \neq 0,(t, x) \in R^{1} \times R^{n},
$$

then the classical solution of (2.5) must be for blow-up in finite time in $C^{1}\left(0, T ; H^{6}(\Omega)\right)$. [4])

(As positive integer $k=1$, then it is theorem 3.2 in

Proof. Since $f(u)=F\left(|u|^{2}\right) u,(c>0)$, we have that

$$
|f(\lambda)|=\left|F\left(|\lambda|^{2}\right) \lambda\right| \geq c|\lambda|^{4 k-1},
$$

and

$$
\bar{\lambda} f(\lambda)=\bar{\lambda} c \lambda|\lambda|^{2}=c|\lambda|^{4 k} \geq 0 .
$$

Thus, from theorem 2.1, we complete the proof of theorem 2.3. (As $k=1$, it is theorem 3.3 in [4])

Now, we may consider the following problem:

$$
\left\{\begin{array}{l}
u_{t}+\mathrm{i} \Delta^{3} u=k\left(\mathrm{e}^{p||^{2}}-1\right) u, t>0, x \in \Omega, \\
u(0, x)=\phi(x), x \in \Omega, \\
{\left.\left[\alpha(t, x)\left(\partial \Delta^{2} u / \partial \gamma\right)+\beta(t, x) u\right]\right|_{\partial \Omega}=0, t \geq 0 .}
\end{array}\right.
$$

where constant $k>0, p>0$.

Theorem 2.4. Assume that $\phi(x) \in H^{6}(\Omega)$, and $\phi(x) \neq 0$, then the solution of (2.6) must be for blow-up in finite time in $C^{1}\left(0, T ; H^{6}(\Omega)\right)$.

Proof. From

$$
\mathrm{e}^{p|u|^{2}}=1+p|u|^{2}+\frac{1}{2 !}\left(p|u|^{2}\right)^{2}+\cdots,
$$

then $f(u)=K\left(\mathrm{e}^{p|u|^{2}}-1\right) u$ satisfy $\bar{\lambda} f(\lambda) \geq 0$, and

$$
|f(\lambda)|>k p|\lambda|^{4 k-1} \text {. }
$$

It holds the condition of theorem 2.1, then by theorem 2.1 that we know the solution of problem (2.6) must be blow-up in finite time. Therefore, we complete the proof of theorem 2.4.

\section{Main Results}

We consider the initial boundary value of some higher order nonlinear Schrodinger equation. By using of eigenfunction method, we can get new results bellow.

Let

$$
\begin{aligned}
& D u=\left(u_{t}, D_{x} u\right)=\left(u_{t}, u_{x_{1}}, u_{x_{2}} \cdots, u_{x_{n}}\right), \\
& (D u)_{x_{i}}=\left(u_{t x_{i}}, u_{x_{1} x_{i}}, \cdots, u_{x_{x} x_{i}}\right),(i=1,2, \cdots, n), \\
& D_{x} D u=\left((D u)_{x_{1}},(D u)_{x_{2}}, \cdots,(D u)_{x_{n}}\right) .
\end{aligned}
$$

Furthermore, we will consider eight-order nonlinear Schrodinger equation. In first, stating that lemma 3.1.

Lemma 3.1. This Eigen-value problem (see [4])

$$
\left\{\begin{array}{l}
\Delta \phi+\lambda \phi=0, x \in \Omega, \\
\left.\partial \phi\right|_{\partial \Omega}=0 .
\end{array}\right.
$$

As we all know the first Eigen valu1e $\lambda_{1} \geq 0$ of $(*)$, the corresponding Eigen-function $\phi_{1}(x)>0$, assume it with

$$
\int_{\Omega} \phi_{1}(x) \mathrm{d} x=1 .
$$

Let $\Omega$ be bounded closed domain in $R^{n}$ and by suite smooth conditions of function $f$ and $g$ that from Green's second formula, we easy get following results.

Now, we consider nonlinear Schrodinger equation with eight-order case $\left(0<\beta_{i}<1, i=1,2\right)$ :

$$
\begin{aligned}
& u_{t}-\mathrm{i}\left(\Delta u+\beta_{1} \Delta^{4} u+\beta_{2} \Delta^{3} u\right) \\
& =f\left(u, D_{x} u, D_{x}^{2} u\right) \\
& +\beta_{1} \Delta^{4} g(u)+\beta_{2} \Delta^{3} g(u) \\
& +\Delta g(u), t>0, x \in \Omega, \\
& u(x, 0)=u_{0}(x), x \in \Omega, \\
& \left.u\right|_{\partial \Omega}=0, x \in \partial \Omega, t>0 .
\end{aligned}
$$

Clearly, $\beta_{1}=\beta_{2}=0$, that is theorem 2.1 in [5].

Theorem 3.1. Assume that problem (3.1)-(3.3) satisfy (where $n$-out normal direction):

$$
\begin{aligned}
& \text { (I) }\left.\frac{\partial G}{\partial n}\right|_{\partial \Omega}=G(0)=0, \\
& G=\operatorname{Re} \beta_{1} \Delta^{3} g(u)+\beta_{2} \operatorname{Re} \Delta^{2} g(u)+g(u) \\
& -\left(\beta_{1} \Delta^{3}+\beta_{2} \Delta^{2}+1\right) \operatorname{Im} u ; \\
& (I I) A=\operatorname{Re} f_{1}\left(u, v, D_{x} u, D_{x}^{2} u\right)+\beta_{1} \lambda^{4} g(u) \\
& \quad-\beta_{2} \lambda^{3} g(u)+\operatorname{Im} u-C F(\operatorname{Re} u), \\
& \alpha=\int_{\Omega} \phi \operatorname{Re} u_{0} \mathrm{~d} x, A \cdot \alpha \geq 0 .
\end{aligned}
$$

$F(u)$ be continuous, convex and even function, here

$$
\begin{aligned}
& C=\left\{\begin{array}{l}
1,(\alpha>0) \\
-1,(\alpha<0)
\end{array} ;\right. \\
& (I I I) F(s)>0(s>\alpha) \text {, and } \int_{\alpha}^{\infty} \mathrm{d} s / F(s)<\infty .
\end{aligned}
$$

Then the classical solution of (4.1)-(4.3) must be blowup in finite time.

Proof. (I) step, when $A \geq 0, \alpha>0$ and $A \cdot \alpha \geq 0$. In the similar way by [5] from that (4.1) first we take the real part of both sides for (4.1), we get that 


$$
\begin{aligned}
& \operatorname{Re} u_{t}-\operatorname{Re}\left(\beta_{1} \Delta^{4} u+\beta_{2} \Delta^{3} u+\Delta u\right) \\
& =\operatorname{Re} f+\operatorname{Re}\left(\beta_{1} \Delta^{4} g(u)+\beta_{2} \Delta^{3} g(u)+\Delta g(u)\right), \\
& \operatorname{Re} u_{t}-\left(\beta_{2} \Delta^{4} \operatorname{Im} u+\beta_{1} \Delta^{3} \operatorname{Im} u+\Delta \operatorname{Im} u\right) \\
& =\operatorname{Re} f\left(u, D_{x} u, D_{x}^{2} u\right) \\
& +\operatorname{Re}\left(\beta_{2} \Delta^{4} g(u)+\beta_{1} \Delta^{3} g(u)+\Delta g(u)\right) .
\end{aligned}
$$

Multiplying by $\phi(x)$ the both sides of (3.4) and integral on $\Omega$ for $x$, it is form:

$\int_{\Omega} \phi \operatorname{Re} u_{t} \mathrm{~d} x=\int_{\Omega} \phi\left[-\left(\beta_{1} \Delta^{4}+\beta_{2} \Delta^{3}+\Delta\right) \operatorname{Im} u\right.$

$\left.+\operatorname{Re} f\left(u, D_{x} u, D_{x}^{2} u\right)+\left(\beta_{1} \Delta^{4}+\beta_{2} \Delta^{3}+\Delta\right) \operatorname{Re} g(u)\right] \mathrm{d} x$

Taking $a(t)=\int_{\Omega} \phi \operatorname{Re} u \mathrm{~d} x$, then $a^{\prime}(t)=\int_{\Omega} \varphi \operatorname{Re} u_{t} \mathrm{~d} x$, and that

$$
\begin{aligned}
a^{\prime}(t)= & \int_{\Omega} \phi\left[-\left(\beta_{1} \Delta^{4}+\beta_{2} \Delta^{3}+\Delta\right) \operatorname{Im} u\right. \\
& +\operatorname{Re} f\left(u, D_{x} u, D_{x}^{2} u\right) \\
& \left.+\left(\beta_{1} \Delta^{4}+\beta_{2} \Delta^{3}+\Delta\right) \operatorname{Re} g(u)\right] \mathrm{d} x \\
= & \int_{\Omega}[\phi \cdot \Delta G-\Delta \phi \cdot(G)] \mathrm{d} x \\
& +\int_{\Omega}\left[\phi \operatorname{Re} f\left(u, D_{x} u, D_{x}^{2} u\right)\right] \mathrm{d} x \\
& -\int_{\Omega}\left[\Delta \phi\left(\beta_{1} \Delta^{3}+\beta_{2} \Delta^{2}+1\right) \operatorname{Im} u\right] \mathrm{d} x \\
& -\int_{\Omega}\left[\left(\beta_{1} \Delta^{3}+\beta_{2} \Delta^{2}+1\right) \operatorname{Re} g(u)\right] \mathrm{d} x
\end{aligned}
$$

By $\left.(\partial G / \partial n)\right|_{\partial \Omega}=0$ in $(I)$ and Green's second formula:

$$
\int_{\Omega}(\phi \Delta G-G \Delta \phi) \mathrm{d} x=0,
$$

Substituting (3.6) into (3.5), we get

$$
\begin{aligned}
& a^{\prime}(t)=\int_{\Omega}\left[\phi \operatorname{Re} f\left(u, D_{x} u, D_{x}^{2} u\right)\right. \\
& -\Delta \phi\left(\left(\beta_{1} \Delta^{3}+\beta_{2} \Delta^{2}+\Delta\right) \operatorname{Im} u\right. \\
& \left.\left.-\left(\beta_{1} \Delta^{3}+\beta_{2} \Delta^{2}+\Delta\right) \operatorname{Re} g(u)\right)\right] \mathrm{d} x \\
& =\int_{\Omega} \phi\left[\operatorname{Re} f\left(u, D_{x} u, D_{x}^{2} u\right)+\lambda\left(\beta_{1} \Delta^{3}+\beta_{2} \Delta^{2}+\Delta\right) \operatorname{Im} u\right. \\
& \left.-\lambda\left(\beta_{1} \Delta^{3}+\beta_{2} \Delta^{2}+\Delta\right) \operatorname{Re} g(u)\right] \mathrm{d} x
\end{aligned}
$$

Hence,

$$
\begin{aligned}
a^{\prime}(t) & =\int_{\Omega} \phi\left[\operatorname{Re} f\left(u, D_{x} u, D_{x}^{2} u\right)\right. \\
& +\left(\beta_{1} \lambda^{4}-\beta_{2} \lambda^{3}-\lambda\right) g(u) \\
& \left.+\left(\left(\beta_{1} \lambda^{4}-\beta_{2} \lambda^{3}-\lambda\right) \operatorname{Im} u\right)\right] \mathrm{d} x
\end{aligned}
$$

From $A \geq 0$,

$$
\begin{aligned}
A= & \operatorname{Re}\left(f\left(u, D_{x} u, D_{x}^{2} u\right)+\left(\beta_{1} \lambda^{4}-\beta_{2} \lambda^{3}-\lambda\right) g(u)\right) \\
& +\left(\beta_{1} \lambda^{4}-\beta_{2} \lambda^{3}-\lambda\right) \operatorname{Im} u-C F(\operatorname{Re} u) \geq 0 .
\end{aligned}
$$

Therefore, we have

$$
\begin{aligned}
& \operatorname{Re}\left(f\left(u, D_{x} u, D_{x}^{2} u\right)+\left(\beta_{1} \lambda^{4}-\beta_{2} \lambda^{3}-\lambda\right) g(u)\right) \\
& \geq\left(-\beta_{1} \lambda^{4}+\beta_{2} \lambda^{3}+\lambda\right) \operatorname{Im} u+C F(\operatorname{Re} u)
\end{aligned}
$$

Combing (3.7)-(3.8), and Jensen's inequality, we obtain

$$
a^{\prime}(t) \geq \phi F(\operatorname{Re} u) \geq F\left(\int_{\Omega} \phi \operatorname{Re} u \mathrm{~d} x\right)=F(a(t))
$$

Here, $F(a(t)) \leq \mathrm{d} a / \mathrm{d} t$. So, $t \leq \int_{\alpha}^{a(t)} \mathrm{d} a /(F(a))$, there exist $T \leq \int_{\alpha}^{\infty} \mathrm{d} a /(F(a))<+\infty$, such that

$$
\lim _{t \rightarrow T} a(t)=+\infty \text {. }
$$

From $a(t)=\int_{\Omega} \phi \operatorname{Re} u \mathrm{~d} x$, and Holder inequality, we get $\left(p^{-1}+q^{-1}=1\right)$,

$$
a(t)=\int_{\Omega} \phi \operatorname{Re} u \mathrm{~d} x \leq\|\phi\|_{L^{q}(\Omega)}\|\operatorname{Re} u\|_{L^{p}(\Omega)},
$$

that is $a(t)\left(\|\phi\|_{L^{q}(\Omega)}\right)^{-1} \leq\|\operatorname{Re} u\|_{L^{p}(\Omega)}$.

Therefore,

$$
\lim _{t \rightarrow T} a(t)\left(\|\phi\|_{L^{q}(\Omega)}\right)^{-1} \leq \lim _{t \rightarrow T}\|\operatorname{Re} u\|_{L^{p}(\Omega)} .
$$

Hence,

$$
\lim _{t \rightarrow T}\|\operatorname{Re} u\|_{L^{p}(\Omega)}=+\infty, \forall 1 \leq p \leq+\infty .
$$

(II) step, when $A \leq 0, \alpha<0$, taking that

$$
u(x, t)=-u_{1}(x, t) \text {, }
$$

then $\operatorname{Re} u=-\operatorname{Re} u_{1}$.

Therefore, let $a(t)=\int_{\Omega} \varphi \operatorname{Re}\left(u_{1}\right) \mathrm{d} x$, we have

$$
a_{1}(t)=-a(t), a_{1}^{\prime}(t)=-a^{\prime}(t), \alpha_{1}=-\alpha>0,
$$
that

Combine (4.1)-(4.8) and $A \leq 0,(C=-1)$, we obtain

$$
-a_{1}^{\prime}(t) \leq-\int_{\Omega} \varphi F\left(-\operatorname{Re} u_{1}\right) \mathrm{d} x
$$

That is also $a_{1}^{\prime}(t) \geq \int_{\Omega} \varphi F\left(-\operatorname{Re} u_{1}\right) \mathrm{d} x$. From Jensen inequality and $F(s)$ is even function, we have

$$
F\left(a_{1}\right)=F\left(-a_{1}\right) \leq \mathrm{d} a_{1} / \mathrm{d} t
$$

then

$$
\mathrm{d} t \leq \mathrm{d} a_{1} / F\left(a_{1}\right)
$$

From (3.12) and similar (I)-step, we can get 


$$
\begin{aligned}
& \lim _{t \rightarrow T}\left\|\operatorname{Re} u_{1}\right\|_{L^{p}(\Omega)}=+\infty, \forall 1 \leq p \leq+\infty . \\
& \lim _{t \rightarrow T}\|\operatorname{Re} u(t)\|_{L^{p}(\Omega)}=\lim _{t \rightarrow T}\|-\operatorname{Re} u(t)\|_{L^{p}(\Omega)}=+\infty, \\
& \forall 1 \leq p \leq+\infty .
\end{aligned}
$$
3.1 .

Combine (I)-(II) we complete the proof of theorem Clearly, $\beta_{1}=\beta_{2}=0$, that is theorem 2.1 in [5].

Theorem 3.2. Assume that problem (3.1)-(3.3) satisfy:

$$
\begin{gathered}
\left.(I) \frac{\partial G}{\partial n}\right|_{\partial \Omega}=G(0)=0, \\
G=\left(\beta_{1} \Delta^{3}+\beta_{2} \Delta^{2}+1\right) \operatorname{Re} g(u) \\
-\left(\beta_{1} \Delta^{3}+\beta_{2} \Delta^{2}+1\right) \operatorname{Im} u ; \\
(I I) B=\frac{\operatorname{Im}\left(f\left(u, D_{x} u, D_{x}^{2} u\right)-\left(\beta_{1} \lambda^{3}-\beta_{2} \lambda^{2}+\lambda\right) g(u)\right)}{F(\operatorname{Im} u)} \\
+\frac{\left(\beta_{1} \lambda^{3}-\beta_{2} \lambda^{2}+\lambda\right)(\operatorname{Re} u)}{F(\operatorname{Im} u)},
\end{gathered}
$$

and

$$
|B|-1 \geq 0, \beta=\int_{\Omega} \phi \operatorname{Im} u_{0} \mathrm{~d} x<0,
$$

where $F(s)$ is continuous, convex and even function;

$$
\text { (III) } F(s)>0,(s>\beta) \text { and } \int_{\beta}^{+\infty} \mathrm{d} s / F(s)<+\infty \text {. }
$$

Then the classical solution for this problem (3.1)-(3.3) is blow-up in finite time.

Proof. From $|B|-1 \geq 0$, we discuss two case:

$$
(I) B-1 \geq 0, \beta<0, u(x, t)=\mathrm{i} \bar{u}_{2}(x, t),
$$

then $\operatorname{Im} u=\operatorname{Re} \bar{u}_{2}$.

Taking the imaginary part for both sides of (3.1), similar the method of proof for Theorem 3.1, we can easy have

$$
\lim _{t \rightarrow T}\left\|\operatorname{Re} u_{2}\right\|_{L^{p}(\Omega)}=+\infty, \forall 1 \leq p \leq+\infty .
$$

So, we get that

$$
\lim _{t \rightarrow T}\|\operatorname{Im} u(t)\|_{L^{p}(\Omega)}=+\infty, \forall 1 \leq p \leq+\infty .
$$

(II) $B+1 \leq 0, \beta<0$, we may let $u(x, t)=-\bar{u}_{3}(x, t)$, then $\operatorname{Im} u=\operatorname{Im} u_{3}$.

$$
\text { So, } \lim _{t \rightarrow T}\left\|\operatorname{Im} u_{3}(t)\right\|_{L^{p}(\Omega)}=+\infty, \forall 1 \leq p \leq+\infty \text {. }
$$

Taking the imaginary part for both sides of (1), by (II) and similar the method of proof for theorem 3.1, we can easy have

$$
\lim _{t \rightarrow T}\left\|\operatorname{Re} u_{3}\right\|_{L^{p}(\Omega)}=+\infty, \forall 1 \leq p \leq+\infty .
$$

We get that

$$
\lim _{t \rightarrow T}\|\operatorname{Im} u(t)\|_{L^{p}(\Omega)}=+\infty, \forall 1 \leq p \leq+\infty .
$$

Combine $(I)-(I I)$, we complete the proof of theorem 3.2 .

Corollary 3.3. Clearly $\beta_{1}=\beta_{2}=0$, that is theorem 2.2 in [5]. By ([13] )looking it for some applications.

\section{Some Higher-Order Case}

In the same way, we can consider the higher-order case (integer $k>0$ ):

$$
\begin{aligned}
& u_{t}-\mathrm{i}\left(\Delta u+\beta_{1} \Delta^{4 k} u+\beta_{2} \Delta^{4 k-1} u\right) \\
& =f\left(u, D_{x} u, D_{x}^{2} u\right)+\beta_{1} \Delta^{4 k} g(u) \\
& +\beta_{2} \Delta^{4 k-1} g(u)+\Delta g(u), t>0, x \in \Omega, \\
& u(x, 0)=u_{0}(x), x \in \Omega \\
& \left.u\right|_{\partial \Omega}=0, x \in \partial \Omega, t>0 .
\end{aligned}
$$

Clearly, $k=1$, that is problem of eight order case.

Theorem 4.1. Assume that problem (4.1)-(4.3) satisfy

(I) $\left.\frac{\partial G}{\partial n}\right|_{\partial \Omega}=G(0)=0, P_{2 k}(\Delta)=\left(\beta_{1} \Delta^{4 k}+\beta_{2} \Delta^{4 k-1}+\Delta\right)$,

$$
\text { and } G=P_{4 k}(\Delta) \operatorname{Re} g(u)-P_{4 k}(\Delta) \operatorname{Im} u \text {; }
$$

(II) $B=$

$$
\frac{\operatorname{Im}\left(f\left(u, D_{x} u, D_{x}^{2} u\right)-P_{4 k}(\lambda) g(u)\right)+P_{4 k}(\lambda)(\operatorname{Re} u)}{F(\operatorname{Im} u)}
$$

and

$$
|B|-1 \geq 0, \beta=\int_{\Omega} \phi \operatorname{Im} u_{0} \mathrm{~d} x<0,
$$

where $F(s)$ is continuous, convex and even function;

$$
\text { (III) } F(s)>0,(s>\beta) \text { and } \int_{\beta}^{+\infty} \mathrm{d} s / F(s)<+\infty .
$$

Then the classical solution for this problem (4.1)-(4.3) is blow-up in finite time.(omit this similar proof)

Remark 4.2. Assume that (here $4 k>3$ )

$$
P_{4 k}(\Delta)=\left(\beta_{1} \Delta^{4 k}+\beta_{2} \Delta^{4 k-1}+\beta_{3} \Delta^{4 k-2}+\beta_{4} \Delta^{4 k-3}\right),
$$

then we will obtain similar results of theorem 3.2 with more case.

Remark 4.3. (See $[6,14]$ ) According to the direction of [6], we may consider that coupled nonlinear Schrodinger equation as in the following iterative formulas in an algorithmic form by VIM:

$$
\begin{aligned}
& A_{n+1}(x, t)=A_{n}(x, t) \\
& -\int_{0}^{t}\left[A_{n_{t}}-\mathrm{i} A_{n_{x x}}-\mathrm{i}\left(\left|A_{n}\right|^{2}+\beta\left|B_{n}\right|^{2}\right) A_{n}\right] \mathrm{d} \tau,
\end{aligned}
$$




$$
\begin{aligned}
& B_{n+1}(x, t)=B_{n}(x, t) \\
& -\int_{0}^{t}\left[B_{n_{t}}-\mathrm{i} B_{n_{x x}}-\mathrm{i}\left(\beta\left|A_{n}\right|^{2}+\left|B_{n}\right|^{2}\right) B_{n}\right] \mathrm{d} \tau .
\end{aligned}
$$

The solution procedure with initial approximations (omit the details ):

$$
\begin{aligned}
& A_{0}=A(x, 0)=f(x)=a(1-\varepsilon \cos (\alpha x)), \\
& B_{0}=B(x, 0)=g(x)=b_{0}(1-\varepsilon \cos (\alpha x)) .
\end{aligned}
$$

The other components can be obtained directly:

$$
A_{1}(x, t), B_{1}(x, t), \cdots, \text { etc. }
$$

Furthermore, the conserved quantities:

$$
E(A)=\int_{-s / 2}^{s / 2}|A(x, t)|^{2} \mathrm{~d} x
$$

and

$$
E(B)=\int_{-s / 2}^{s / 2}|B(x, t)|^{2} \mathrm{~d} x,
$$

where $s=2 \pi / \alpha$. This numerical results is with higher accuracy.

\section{The Global Attractor of the Fractional NSE}

Recently, they also showed that dynamic behavior of large time action to investigate for $[15,16]$, they are deepgoing study global attractor and dimension estimate of integer order non-linear Schrodinger equation in [16].

The author search the Cauchy problem for fractional order non-linear Schrodinger equation in [17]. The author search the global attractor problem for a class of fractional order non-linear Schrodinger equation in [17] and we based on [16-18], and combine [19] obtained the condition of existence of solution for following fractional order non-linear Schrodinger equation:

$$
\left\{\begin{array}{l}
\mathrm{i} u_{t}+(-\Delta)^{\alpha} u+\beta F\left(|u|^{p} u\right)+\mathrm{i} \delta u \\
=f(x), x \in \Omega, t>0, \\
u(x, 0)=u_{0}(x), x \in \Omega, \\
u\left(x+L e_{i}\right), t=u(x, t), x \in \Omega, t>0 .
\end{array}\right.
$$

Physics background of (1) is arise the main part of nonlinear interaction for laser and plasma, $u$ express the field of electricity [20], where

$$
\Omega=(0, l)^{n}, e_{i}=(0, \cdots, 0,1,0, \cdots, 0), i=1,2, \cdots, n
$$

is with standard perpendicular base, $i$ is imaginary unit, the function $F(\eta)$ is with one order derivative $\alpha>(n / 2), \beta>0, p>0$, where $\delta>0$ with some consume effect, and as $\delta=0$ express the integral system with soliton solution.

As $\alpha=3, \beta=-c, \delta=1$ for (3.1), and (4.1) thirdly section case, we will obtain global attractor of initial value problem (5.1) that first give out Lemma as follows.

Lemma 5.1. Let

$$
u_{0}(x) \in L^{2}(\Omega), f(x) \in L^{2}(\Omega), u(x, t)
$$

is the solution of problem (5.1), and

$$
\|u(x, t)\|^{2} \leq\left\|u_{0}(x)\right\|^{2} \mathrm{e}^{-\delta t}+(\delta)^{-2}\|f(x)\|^{2} .
$$

Proof. Multiply $\bar{u}$ for the both sides of (**) act as inner product, we have

$$
\begin{aligned}
& \left(\mathrm{i} u_{t}, \bar{u}\right)+\left((-\Delta)^{\alpha} u, \bar{u}\right) \\
& +\left(\beta F\left(|u|^{p} u\right), \bar{u}\right)+(\mathrm{i} \delta u, \bar{u}) \\
& =(f(x), \bar{u})
\end{aligned}
$$

and take real part,

$$
\begin{aligned}
& 2^{-1} \mathrm{~d}\left(\|u\|^{2} / \mathrm{d} t\right)+\delta\|u\|^{2} \\
& =\operatorname{Im}(f(x), \bar{u}) \leq\|f(x)\|\|u\| \\
& \leq 2^{-1} \delta\|u\|^{2}+(2 \delta)^{-1}\|f(x)\|^{2},
\end{aligned}
$$

From (5.3) and by use of Gronwall inequality, we obtain

$$
\|u(x, t)\|^{2} \leq\left\|u_{0}(x)\right\|^{2} \mathrm{e}^{-\delta t}+(\delta)^{-2}\|f(x)\|^{2} .
$$

\section{Lemma 5.2. Let}

$$
u_{0}(x) \in H^{\alpha}(\Omega) \cap L^{p+2}(\Omega), f(x) \in H^{\alpha}(\Omega), u(x, t)
$$

is the solution of problem (1), then $\left\|(-\Delta)^{\alpha / 2} u\right\|,\|u\|_{L^{p+2}(\Omega)}$ with uniform bounded.

Proof. To establish inner product for both sides of equation (5.1) with for $\bar{u}_{t}$, and take real part, we have that

$$
\begin{aligned}
& 2^{-1}\left(\left\|(-\Delta)^{\alpha / 2} u\right\|^{2}\right)_{t}^{\prime}+\beta \int_{\Omega} F\left(|u|^{p} u\right) \bar{u}_{t} \mathrm{~d} x-\operatorname{Im}\left(\delta u, \bar{u}_{t}\right) \\
& =\operatorname{Re}\left(f(x), \bar{u}_{t}\right),
\end{aligned}
$$

easy get that by (5.1),

$$
\begin{aligned}
-\operatorname{Im}\left(\delta u, \bar{u}_{t}\right) & =\delta\left\|(-\Delta)^{\alpha / 2} u\right\|^{2} \\
& +\delta \beta \int_{\Omega} F\left(|u|^{p} u\right) \bar{u} \mathrm{~d} x \\
& -\operatorname{Re}\left(f(x), \bar{u}_{t}\right),
\end{aligned}
$$

where

$$
\begin{aligned}
\operatorname{Re}\left(f(x), \bar{u}_{t}\right) & =\operatorname{Im}\left(f(x),(-\Delta)^{\alpha} \bar{u}+\beta F\left(|u|^{p} \bar{u}\right)\right) \\
& -\operatorname{Re}(f(x), \delta \bar{u})
\end{aligned}
$$

by use of Jensen's inequality, we have 


$$
\begin{aligned}
& \left(2^{-1}\left(\left\|(-\Delta)^{\alpha / 2} u\right\|^{2}\right)_{t}^{\prime}+\beta \int_{\Omega} F_{1}\left(|u|^{p} u\right)\left(|u|^{p} u\right) \bar{u}_{t} \mathrm{~d} x\right) \\
& +\delta\left\|(-\Delta)^{\alpha / 2} u\right\|^{2}+\delta \beta \int_{\Omega} F_{1}\left(|u|^{p} u\right)\left(|u|^{p} u\right) \bar{u} \mathrm{~d} x \\
& =\operatorname{Im}\left(f(x),(-\Delta)^{\alpha} \bar{u}+\beta F\left(|u|^{p} \bar{u}\right)\right) \\
& \leq\left\|(-\Delta)^{\alpha / 2} f(x)\right\|\left\|(-\Delta)^{\alpha / 2} u\right\| \\
& +\beta\|f(x)\|_{L^{\infty}}\left(\Omega, L^{2}(\Omega)\right)^{2} F\left(\|u\|_{L^{P+1}}^{P+1}\right) \cdot 2^{-1}\left(\left\|u_{t}\right\|^{2}\right)_{t}^{\prime}+\delta\left\|_{t}\right\|^{2} \\
& =-\operatorname{Im}\left(\beta F\left(|u|^{p} u\right), \bar{u}_{t}\right) \leq \beta \int_{\Omega} F\left(|u|^{p} u\right) \bar{u}_{t} \mathrm{~d} x \\
& \leq 2^{-1} \beta F^{2}\left(\int_{\Omega}|u|^{p} u \mathrm{~d} x\right)+2^{-1} \beta \int_{\Omega}\left(\left|u_{t}\right|^{2}\right) \mathrm{d} x \\
& \leq 2^{-1} \beta F^{2}\left(\|u\|_{L^{P+1}(\Omega)}^{P+1}\right) \\
& +2^{-1} \beta\left\|u_{t}\right\|^{2},\left(\left\|u_{t}\right\|^{2}\right)_{t}^{\prime}+(2 \delta-\beta)\left\|u_{t}\right\|^{2} \leq \beta F^{2}\left(\|u\|_{L^{P+1}(\Omega)}^{P+1}\right) .
\end{aligned}
$$

So,

$$
\begin{aligned}
& \left(2^{-1}\left(\left\|(-\Delta)^{\alpha / 2} u\right\|^{2}\right)_{t}^{\prime}+\beta \int_{\Omega} F\left(|u|^{p} u\right) \bar{u}_{t} \mathrm{~d} x\right) \\
& \leq 2 \delta\left(2^{-1}\left\|(-\Delta)^{\alpha / 2} u\right\|^{2}+\beta \int_{\Omega} F\left(|u|^{p} u\right) \bar{u} \mathrm{~d} x\right)+C
\end{aligned}
$$

by use of Gronwall inequality, we obtain

$$
\left\|(-\Delta)^{\alpha / 2} u\right\|, \int_{\Omega} F\left(|u|^{p} u\right) \bar{u}_{t} \mathrm{~d} x
$$

uniform boundary.

Lemma 5.3. Let

$$
u_{0}(x) \in H^{2 \alpha}(\Omega), f(x) \in L^{2}(\Omega), u(x, t)
$$

is the solution of problem (5.1), then $\left\|(-\Delta)^{\alpha} u\right\|$ with uniform bounded.

Proof. To derivative both sides of Equation (5.1) for $t$ and take inner product for $\bar{u}_{t}$, and taking also imaginary part, we have

$$
2^{-1}\left(\left\|u_{t}\right\|^{2}\right)_{t}^{\prime}+\operatorname{Im}\left(\beta F\left(|u|^{p} u\right), \bar{u}_{t}\right)+\delta\left\|u_{t}\right\|^{2}=0
$$

Then

$$
\begin{aligned}
& 2^{-1}\left(\left\|u_{t}\right\|^{2}\right)_{t}^{\prime}+\delta\left\|u_{t}\right\|^{2} \\
& =-\operatorname{Im}\left(\beta F\left(|u|^{p} u\right), \bar{u}_{t}\right) \\
& \leq 2^{-1} \beta\left(F^{2}\left(\|u\|_{L^{\infty}(\Omega)}^{P+1}\right)+\left\|u_{t}\right\|^{2}\right), \\
& \left(\left\|u_{t}\right\|^{2}\right)_{t}^{\prime}+(2 \delta-\beta)\left\|u_{t}\right\|^{2} \leq \beta F^{2}\left(\|u\|_{L^{\infty}(\Omega)}^{P+1}\right)
\end{aligned}
$$

By Lemma 5.2 and Young inequality, the (5.4) with form

$$
\left(\left\|u_{t}\right\|^{2}\right)_{t}^{\prime}+2 \delta\left\|u_{t}\right\|^{2} \leq \delta\left\|u_{t}\right\|^{2}+C
$$

by use of Gronwall inequality, we obtain

$$
\left\|u_{t}\right\|^{2} \leq\left\|u_{0}(x, 0)\right\|^{2} \mathrm{e}^{-\delta t}+\delta^{-1} C
$$

Because hold these inequality bellow

$$
\begin{aligned}
& \left\|u_{t}(x, 0)\right\| \leq C\left(\left\|(-\Delta)^{\alpha} u_{0}\right\|+\beta F\left(\left|u_{0}\right|^{p} u_{0}\right)+\delta\left\|u_{0}\right\|\right) \\
& \leq C\left\|u_{0}\right\|_{H^{2 \alpha}(\Omega)} \\
& \left\|(-\Delta)^{\alpha} u_{0}\right\| \leq\left\|u_{t}\right\|+\beta F\left(|u|^{p} u\right)+\delta\|u\| \\
& \leq\left\|u_{t}\right\|+\beta F\left(\|u\|_{L^{\infty}(\Omega)}^{P-1}\|u\|^{2}\right)+\delta\|u\|,
\end{aligned}
$$

Hence $\left\|u_{t}\right\|,\left\|(-\Delta)^{\alpha} u\right\|$ are uniform boundary. Similar method of $[19,20]$, we give out that condition of yield global attractor of problem (5.1).

Theorem 5.4. Assume hat

$$
\begin{aligned}
& u_{0}(x) \in H^{2 \alpha}(\Omega), f(x) \in L^{2}(\Omega), \\
& \alpha>(n / 2), \beta>0, p>0,
\end{aligned}
$$

then the periodic global attractor of initial value problem (4.1-4.3):

$$
A=\bigcap_{s \geq 0} \overline{\bigcup_{t \geq s} S(t) B_{0}}
$$

where $S(t)$ for operator semi-group with needing define in prove and $B_{0}$ for with the bounded attractor set in following in prove processes.

Proof. We omit the proof (by using of similar proof method in $[18,19])$.

Remark 5.5. Furthermore, we shall study global attractor of fraction order non-linear Schrodinger type equation, and the estimate for its dimensions, and that blowing-up of solution for some fraction order non-linear Schrodinger type equation.

\section{Some Notes for Shake Power and Light Power}

We consider some meaning of physic and Energy for nonlinear Schrodinger equation.The numerical test for solution of nonlinear Schrodinger equation with ground state and excite state.

Atoms absorb energy from the ground state transition to the excited state, learned through experiments in extreme case, the ground state solution is not controlled solution-Blow-up solution.

Thus, strictly control the number and perturbation for impulsive velocity of the atomic transition, is one of the 
main methods to produce new material structure. Strict control of the atomic transition to the first, second and third excited state is more practical significance, especially the transition to the first excited state. As we all now, the ultra-low temperatures, the atomic gas in the magnetic potential well Boer-Einstein condensation experiments [21], promotion of scholars study the macroscopic quantum behavior of atoms and kinetic characteristics.

By using of above stating method we consider calculate to the ground state solution and excite state of ddimension BECS (Bose-Einstein condensate) with mix harmonic potential and crystal lattice potential.

The Gross-Pitaevskii equation:

$$
\begin{aligned}
& \mathrm{i} \bar{h}\left(\frac{\partial \psi(r, t)}{\partial t}\right) \\
& =\left(-\left(\frac{\bar{h}^{2}}{2 m}\right) \Delta^{2}+V(r)+N U_{0}|\psi(r, t)|^{2}\right) \psi(r, t)
\end{aligned}
$$

where $r \in R^{d}, d=1,2,3 . t>0, \quad m$ expresses mass of atoms, $\bar{h}$ be planck constant, $N$ be number of atoms in cohesion system, $V(r)$ be outer power,

$U_{0}=\left(4 \pi \bar{h}^{2} a\right) / m$ describe interaction between the atoms cohesion $\left(a_{s}>0\right.$, means repel; $a_{s}<0$, shows attract each other). Thus, by pass appropriate immeasurable process, then the (6.1) may be written:

$$
\mathrm{i} \frac{\partial \psi(r, t)}{\partial t}=\left(-\frac{1}{2} \Delta^{2}+V(r)+\beta|\psi(r, t)|^{2}\right) \psi(r, t)
$$

The parameter $\beta$ for positive, or negative, describe that repel or attract corresponding, out power $V(r)$ be defined by physic system for us to study things. By using of the imaginary time method to calculate it in [22] that let $\tau=$ it substituting it into (6.2), we have

$$
-(\partial \psi / \partial(\tau))=\left(-2^{-1} \Delta+V+\beta|\psi|^{2}\right) \psi
$$

So, by check parameter method in [23] we check nonlinear parameter for light rule power, then we get ground state and excite state correspondingly.

\subsection{One Dimension Case $(d=1)$}

(a) We consider two class powers (shake power and light power) in (6.3), Setting shake power

$$
V(x)=x^{2} / 2, b=250, b_{1}=2
$$

taking initial wave

$$
\varphi_{0}(x)=\sqrt{\frac{2}{\sqrt{\pi}}} \mathrm{e}^{-\left(\frac{b_{1}}{2}\right) x^{2}},
$$

to calculate ground state $\varphi_{g}$. For (6.4) we calculate first arouse state $\varphi_{1}$, space field for $-10<x<10$, the time step for $\Delta t=0.2$.

(b) Similar above way, taking

$$
V(x)=\left(x^{2} / 2\right)+25 \sin ^{2}(\pi x / 4), b=500, b_{1}=2,
$$

and (6.3) for

$$
\varphi_{g}: \varphi_{0}(x)=\sqrt{\frac{2}{\sqrt{\pi}}} x \mathrm{e}^{-\left(\frac{b_{1}}{2}\right) x^{2}},
$$

and $-10<x<10$, and $\Delta t=0.2$.

On the other hand, by the MATLAB search the solution of Equation (6.3) in case (1) and (2) as follow with $\varphi_{0}, \varphi_{1}, \varphi_{2}, \varphi_{3}$ (See Figures 1 and 2).

\subsection{Two-Dimension Case $(d=2)$}

Consider shake power in [14,24]

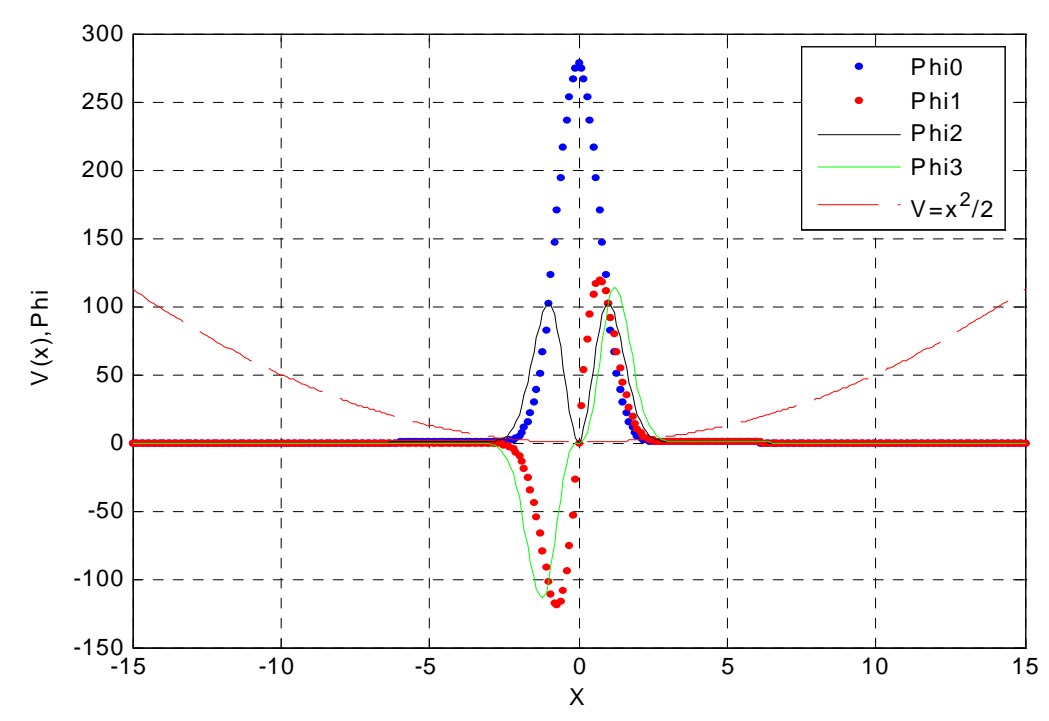

Figure 1. Ground state phi0. First excited state phi1. $V=x^{2} / 2 ; b=500, b i=2$. 


$$
V(x, y)=\left\{\begin{array}{l}
0,0<x<a, 0<y<b \\
\infty, \quad \text { other. }
\end{array}\right.
$$

The grain energy:

$E_{n_{1} n_{2}}=\frac{\pi^{2} \bar{h}^{2}}{2 m}\left(\frac{n_{1}^{2}}{a^{2}}+\frac{n_{1}^{2}}{b^{2}}\right), n_{1}, n_{2}=1,2,3, \cdots ; a=5, b=2$.

We take initial wave function for

$\varphi_{0}(x, y)=\left(\frac{4}{a b}\right)^{1 / 2} \sin \left(\frac{n_{1} \pi x}{a}\right) \sin \left(\frac{n_{2} \pi y}{b}\right), n_{1}=n_{2}=1$.

To calculate ground state $\varphi_{g}$; For

$$
\varphi_{10}(x, y)=\left(\frac{4}{a b}\right)^{1 / 2} x \sin \left(\frac{n_{1} \pi x}{a}\right) \sin \left(\frac{n_{2} \pi y}{b}\right),
$$

$$
\varphi_{01}(x, y)=\left(\frac{4}{a b}\right)^{1 / 2} y \sin \left(\frac{n_{1} \pi x}{a}\right) \sin \left(\frac{n_{2} \pi y}{b}\right),
$$

and

$$
\varphi_{11}(x, y)=\left(\frac{4}{a b}\right)^{1 / 2} x y \sin \left(\frac{n_{1} \pi x}{a}\right) \sin \left(\frac{n_{2} \pi y}{b}\right),
$$

where $n_{1}=n_{2}=2$.

By calculating $\varphi_{10}(x, y)$ along the direction of axe $x$, and $\varphi_{10}(x, y)$ in direction of axe $y$, and calculating first excited of $\varphi_{11}(x, y)$ along direction for axe $x$ and axe $y$, and space field for $0<x<5,0<y<2$, time step: $\Delta t_{x}=0.05, \Delta t_{y}=0.02$.

Combine these cases as Fig: (See Figures 3(a) and (b), Figures 4-6)

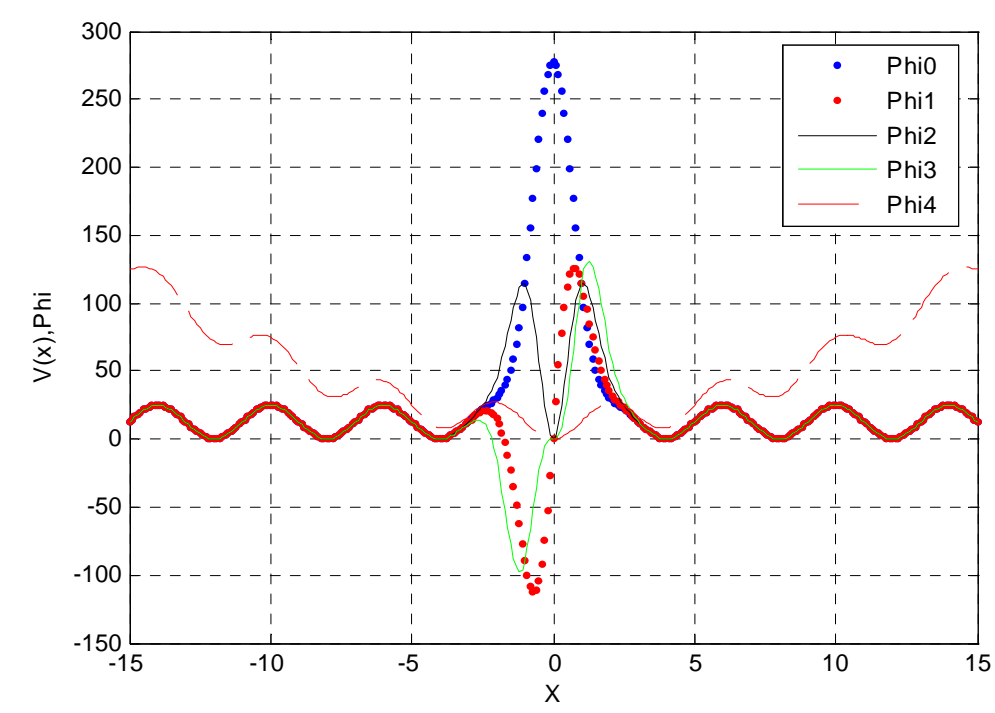

Figure 2. Ground state phi0. First excited state phi1. $V=x^{2} / 2+25 *(\sin (p i * x / 4))^{2} ; b=500, b i=2$.

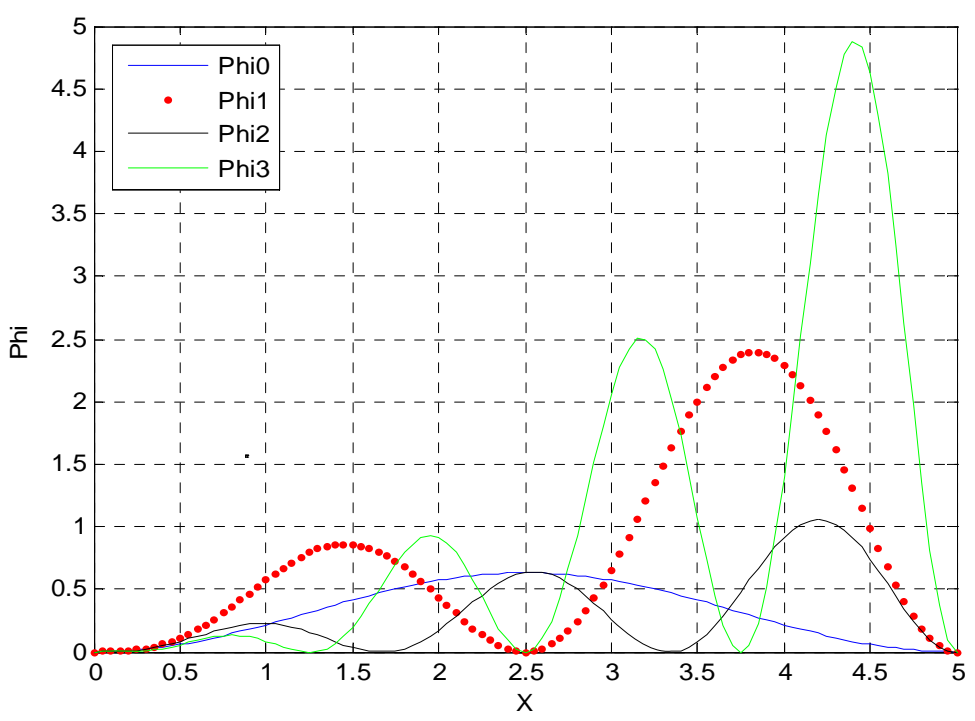

(a) 


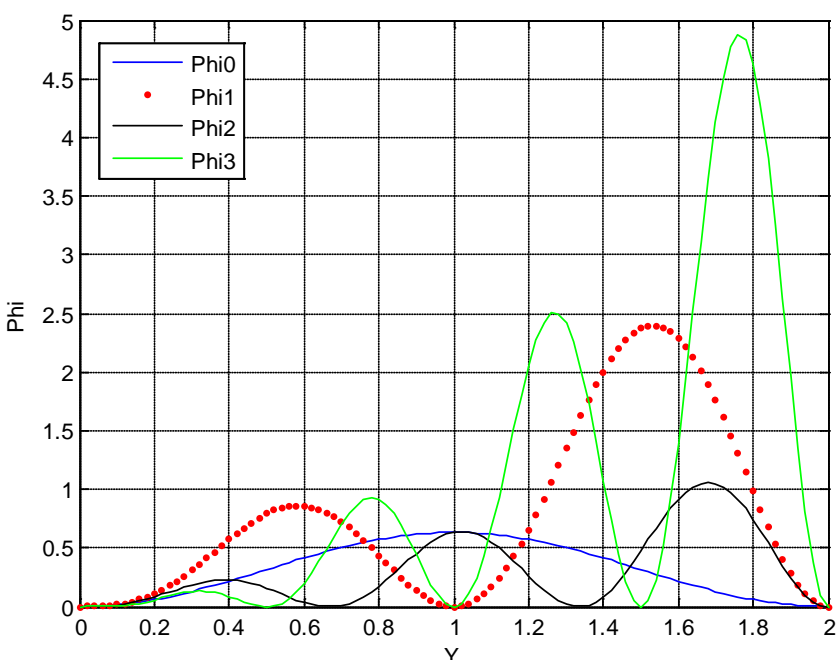

(b)

Figure 3. (a) Ground state phi0. First excited state phi1. $a=5, b=2$. (b) Ground state phi0. First excited state phi1. $a=5, b=$ 2.

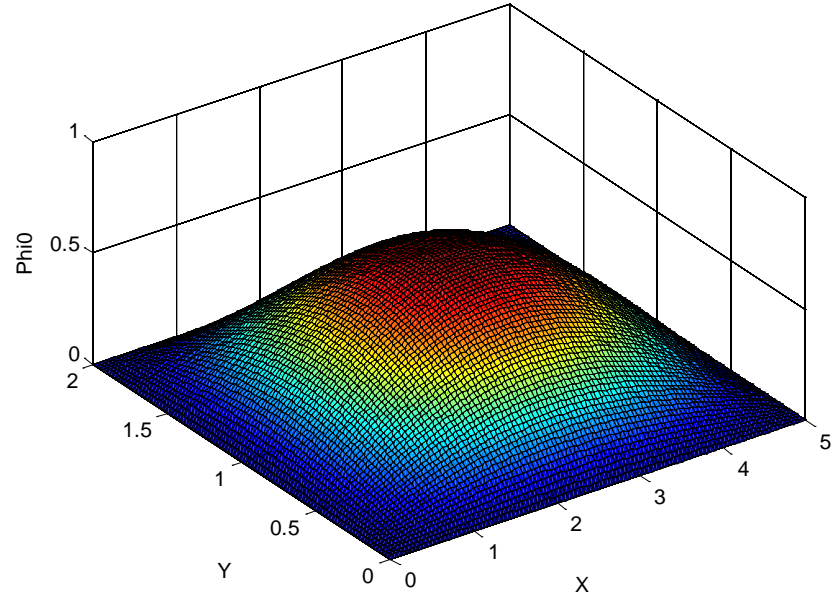

Figure 4. Ground state phi0 $a=5, b=2$.

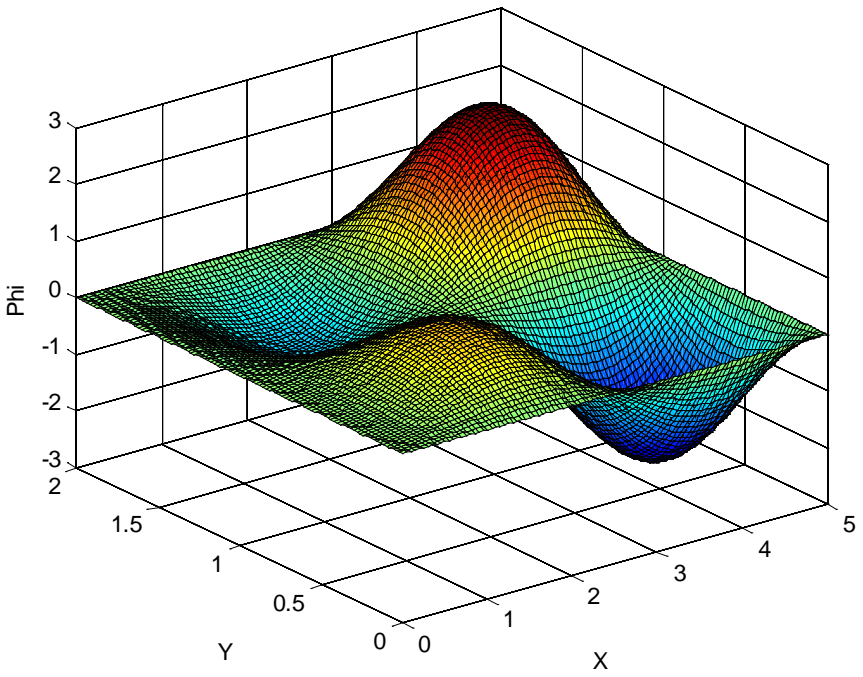

Figure 5. First excited state phi1-x $a=5, b=2$. 


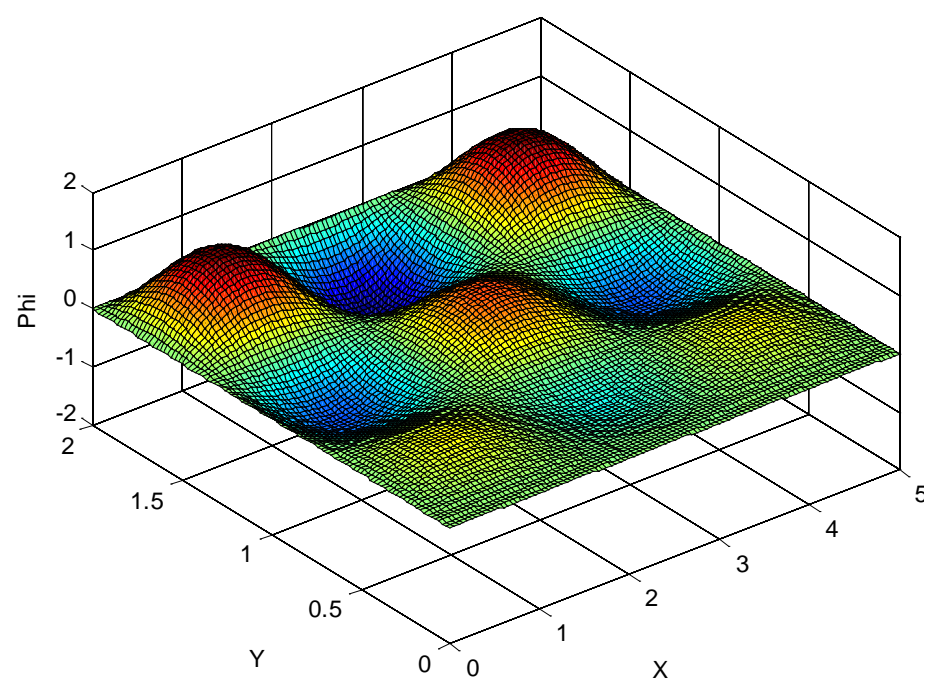

Figure 6. First excited state phi2-y $a=5, b=2$.

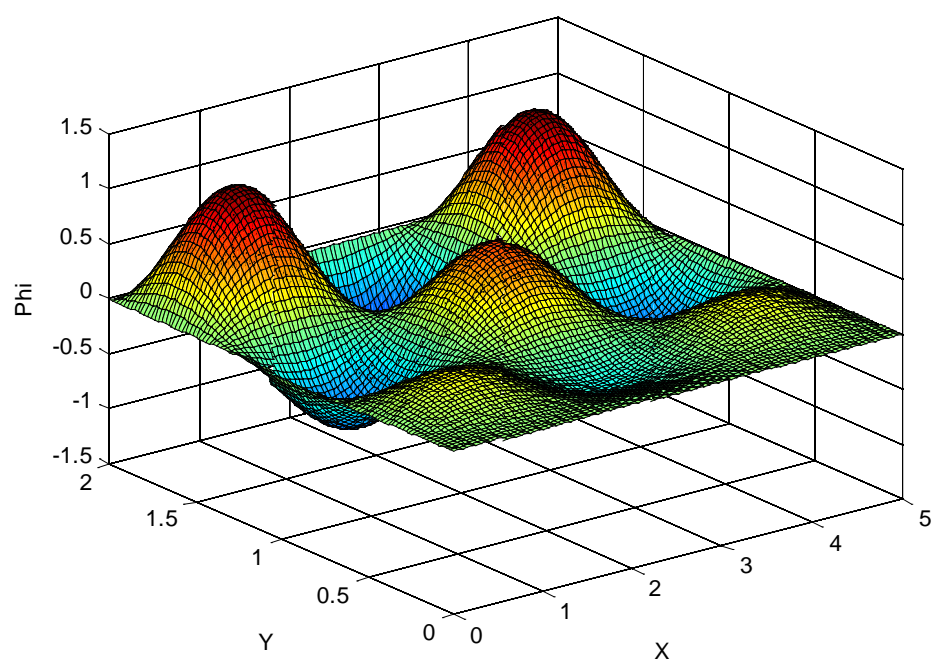

Figure 7. First excited state phi3-xy $a=5, b=2$.

We consider three-dimension case, Figure 4 for ground state $\varphi_{0}(x, y)$ corresponding case, the $\varphi_{10}(x, y)$ as with express along direction of axe $x$ (wave surface) in Figure 5, the $\varphi_{01}(x, y)$ as with express along direction of axe y (wave surface) in Figure 6, the $\varphi_{11}(x, y)$ as for express along direction of axe $x$ and axe $y$ (wave surface) in Figure 7.

\section{Concluding Remarks}

Recently, the higher-order Schrodinger differential equations is also a very interesting topic, and that application of some physics and mechanics of for some more fields as nonlinear Schrodinger equations and some compute methods etc. In our future work, we may obtain some better results.

The application of some physics and mechanics of for some more fields with some combine equations (look [7,
13]).

\section{Acknowledgements}

This work is supported by the Nature Science Foundation (No.11ZB192) of Sichuan Education Bureau (No.11zd 1007 of Southwest University of Science and Technology).

\section{REFERENCES}

[1] B. L. Guo, "Initial Boundary Value Problem for One Class of System of Multi-Dimension Inhomogeneous GBBM Equation,” Chinese Annals of Mathematics, Vol. 8, No. 2, 1987, pp. 226-238.

[2] B. L. Guo and C. X. Miao, "On Inhomogeneous GBBM Equation,” Journal of Partial Differential Equations, Vol. 8, No. 3, 1995, pp. 193-204. 
[3] G. G. Cheng and J. Zhang, "Remark on Global Existence for the Superetitical Nonlinear Shrodinger Equation with a Harmonic Potential,” Journal of Mathematical Analysis and Applications, Vol. 320, No. 2, 2006, pp. 591-598. doi:10.1016/j.jmaa.2005.07.008

[4] J. Zhang, "Blow-Up of Solutions to the Mixed Problems for Nonlinear Schrodinger Equations,” Journal of Sichuan Normal University, Vol. 3, 1989, pp. 1-8.

[5] J. S. Zhao, Q. D. Guo, H. O. Yang and R. Z. Xu, "Blow-Up of Solutions for Initial Value Boundary Problem of a Class of Generalized Non-Linear Schrodinger Equation," Journal of Nature of Science of Heilongjiang University, Vol. 25, No. 2, 2008, pp. 170-172.

[6] N. H. Sweilam and R. F. AI-Bar, "Variational Iteration Method for Coupled Nonlinear Schrodinger Equations," Computers and Mathematics with Applications, Vol. 54, No. 7-8, 2007, pp. 993-999. doi:10.1016/j.camwa.2006.12.068

[7] J. Li and J. Zhan, "Blow-Up for the Stochastic Nonlinear Schrodinger Equation with a Harmonic Potential," Advances in Mathematics, Vol. 39, No. 4, 2010, pp. 491499.

[8] J. Zhang, "Sharp Conditions of Global Existence for Nonlinear Schrodinger and Klein-Golden Equations,” Nonlinear Analysis, Vol. 48, No. 1, 2002, pp. 191-207. doi:10.1016/S0362-546X(00)00180-2

[9] F. Genoud and C. A. Stuart, "Schrodinger Equations with a Spatially Decaying Non-Linear Existence and Stability of Standing Waves," Discrete and Continuous Dynamical Systems, Vol. 21, No. 1, 2008, pp. 137-186.

[10] S. L. Xu, J. C. Liang and L. Yi, "Exact Solution to a Generalized Nonlinear Schrodinger Equation,” Communications in Theoretical Physics, Vol. 53, No. 1, 2010, pp. 159-165.

[11] H. Zhu, Y. Han and J. Zhang, "Blow-Up of Rough Solutions to the Fourth-Order Nonlinear Schrodinger Equation,” Nonlinear Analysts, Vol. 74, No. 17, 2011, pp. 61866201. doi:10.1016/j.na.2011.05.096

[12] H. Meng, B. Tian, T. Xu and H. Q. Zhang, "Backland Transformation and Conservation Laws for the VariableCoefficient N-Coupled Schrodinger Equations with Symbolic Computation,” Acta Mathematica Sinica, Vol. 28, No. 5, 2012, pp. 969-974. doi:10.1007/s10114-011-0531-8

[13] G. R. Jia, J. C. Zhang, X. Z. Hang and Z. Z. Ren, "Coherent Control of Population Transfer in Li Atoms via
Chirped Microwave Pulses," Chinese Physics Letters, Vol. 26, No. 10, 2009, Article ID: 103201-1-4.

[14] N. H. Sweilam and R. F. Ai-Bar, "Variational Iterative Method for Coupled Nonlinear Schrodinger Equations," Computers and Mathematics with Applications, Vol. 54, No. 7-8, 2007, pp. 993-999.

doi:10.1016/j.camwa.2006.12.068

[15] C. S. Zhu, “An Estimate of the Global Attractor for the Non-Linear Schrodinger Equation with Harmonic Potential,” Journal of Southwest Normal university, Vol. 30, No. 5, 2005, pp. 788-791.

[16] B. L. Guo, T. Q. Han and X. N. Jie, "Existence of Global Smooth Solution to the Periodic Boundary of Fractional Non-Linear Schrodinger Equation,” Applied Mathematics and Computation, Vol. 204, No. 1, 2008, pp. 468-477. doi:10.1016/j.amc.2008.07.003

[17] G. G. Lin and H. J. Gao, “Asymptotic Dynamical Difference between the Nonlocal and Local Swift-Hohenberg Models,” Journal Mathematical Physics, Vol. 41, No. 4, 2000, pp. 2077-2089. doi:10.1063/1.533228

[18] L. Wang. J. B. Dang and G. G. Ling, "The Global Attractor of the Fractional Nonlinear Schrodinger Equation and the Estimate of Its Dimension," Journal of Yunnan University, Vol. 32, No. 2, 2010, pp. 130-135.

[19] V. G. Makhankov, "On Stationary Solutions of Schrodinger Equation with a Self-Consistent Satisfying Boussinesq's Equations," Physics Letters A, Vol. 500, 1974, pp. 42-44.

[20] S. Zhang and F. Wang, "Inter Effects between There Coupled Bose-Einstein Condensates," Physics Letters A, Vol. 279, 2001, pp. 231-238.

[21] A. Aftalion and Q. Do, "Vortices in A Rotating BoseEinstein Condensate: Critical Angular Velocities and Energy Diagrams in the Thomas-Fermi regime,” Physics Letters A, Vol. 64, No. 6, 2001, Article ID: 063603. doi:10.1103/PhysRevA.64.063603

[22] C. Tozzo, M. Kramer and F. Dalfovo, "Stability Diagram and Growth Rate of Parametric Resonances in Bose-Einstein Condensates in One Dimensional Optical Lattices," Physics Letters A, Vol. 72, No. 2, 2005, Article ID: 023613. doi:10.1103/PhysRevA.72.023613

[23] J. Y. Zeng, “Quantum Mechanics,” Science Press, Beijing, 2004, pp. 33-144.

[24] R. Teman, "Infinite Dimensional Dynamical Systems in Mechanics and Physics," Springer Verlag, New York, 1988. doi:10.1007/978-1-4684-0313-8 